\title{
Development of a dual-energy silicon $X$-ray diode and its application to gadolinium imaging
}

Yuichi Sato ${ }^{1}$, Eiichi Sato ${ }^{2}$, Shigeru Ehara ${ }^{3}$, Yasuyuki Oda ${ }^{2}$, Osahiko Hagiwara ${ }^{4}$, Hiroshi Matsukiyo $^{4}$, Toshiyuki Enomoto ${ }^{4}$, Manabu Watanabe ${ }^{4}$, Shinya Kusachi ${ }^{4}$

${ }^{1}$ Central Radiation Department, Iwate Medical University Hospital, 19-1 Uchimaru, Morioka, Iwate 020-0023, Japan

${ }^{2}$ Department of Physics, Iwate Medical University, 2-1-1Nishitokuta, Yahaba, Iwate 028-3694, Japan

${ }^{3}$ Department of Radiology, School of Medicine, Iwate Medical University, 19-1 Uchimaru, Morioka, Iwate 020-0023, Japan

${ }^{4}$ Depatment of Surgery, Toho University Ohashi Medical Center, 2-17-6 Ohashi, Meguro, Tokyo 153-8515, Japan

Corresponding author.

E-mail address: dresato@iwate-med.ac.jp

Keywords:

Dual-energy X-ray diode

Double Si diode

Dual-energy CT

Gd-K-edge CT

Energy subtraction 


\section{ABSTRACT}

To perform dual-energy X-ray imaging, we developed a dual-energy silicon X-ray diode (DE-Si-XD) consisting of two ceramic-substrate silicon X-ray diodes (Si-XD) and a 0.2-mm-thick copper filter. The Si-XD is a high-sensitivity Si photodiode selected for detecting X-rays. In the front $\mathrm{Si}-\mathrm{XD}, \mathrm{X}$-ray photons from an X-ray tube are directly detected. Because low-energy photons are absorbed by the front $\mathrm{Si}-\mathrm{XD}$ and the filter, the average photon energy increases when the back $\mathrm{Si}-\mathrm{XD}$ is used. In the front $\mathrm{Si}-\mathrm{XD}$, the photocurrents flowing through the Si-XD are converted into voltages and amplified using current-voltage and voltage-voltage $(\mathrm{V}-\mathrm{V})$ amplifiers. The output from the $\mathrm{V}-\mathrm{V}$ amplifier is input to an analog-digital converter through an integrator for smoothing the voltage. The same amplification method is also used in the back Si-XD. Dual-energy computed tomography (DE-CT) is accomplished by repeated linear scans and rotations of the object, and two projection curves of the object are obtained simultaneously by linear scanning at a tube voltage of $90 \mathrm{kV}$ and a current of $1.0 \mathrm{~mA}$. In the DE-CT, the exposure time for obtaining a tomogram is 10 min with scan steps of $0.5 \mathrm{~mm}$ and rotation steps of $1.0^{\circ}$. Using gadolinium-based contrast media, energy subtraction was performed.

\section{Introduction}

To image 100- $\mu \mathrm{m}$-diameter blood vessels at high contrast, enhanced iodine (I) Kedge angiography (Mori et al., 1996; Hyodo et al., 1998) has been carried out using monochromatic parallel beams with photon energies of approximately $35 \mathrm{keV}$. These photons with energies just beyond I-K-edge energy $33.2 \mathrm{keV}$ are absorbed effectively by I atoms. Successively, a cerium X-ray generator (Sato et al., 2004; Sato et al., 2005) that produces cone beams has been developed to perform I-K-edge imaging because cerium $\mathrm{K} \alpha$ photons with an average energy of $34.6 \mathrm{keV}$ are also absorbed effectively by the I atoms.

I-K-edge imaging can also be carried out by the energy-range selection of $\mathrm{X}$-ray photons. Recently, several photon-counting energy-dispersive computed tomography (ED-CT) systems (Matsukiyo et al., 2011; Hagiwara et al., 2011; Sato et al., 2012) have been developed using a cadmium telluride (CdTe) detector to perform K-edge imaging 
and X-ray fluorescence analysis using I and gadolinium (Gd) media, and a preclinical CT system (Feuerlein et al., 2008; Wang et al., 2011) with a CdTe-array detector has also been developed and applied to K-edge imaging. However, the maximum count rate of a fairly available CdTe detector with an energy resolution of $1 \%$ at $122 \mathrm{keV}$ was approximately 5 kilo-counts per second (kcps) (Shimamura et al., 2014) under the pileup-less condition, and it was difficult to obtain two-different-energy granulation-less tomograms simultaneously.

Although contrast media were observed at high contrast utilizing the K-edge imaging, it is not easy to remove unnecessary regions from tomograms. In view of this situation, the energy-subtraction imaging is useful for imaging only the target region including contrast media. Lately, we have found a high-sensitivity silicon X-ray diode (Si-XD) (Arakawa et al., 2014) that is used to count photons and to determine the maximum photon energy. Using this Si-XD, most X-rays are detected as low-energy photons by Compton scattering from the ceramic substrate behind the $\mathrm{Si}$. Under the pileup condition, the maximum tube current can be increased from $0.1 \mathrm{~mA}$ to $1 \mathrm{~A}$ at a tube voltage of $100 \mathrm{kV}$. Therefore, the single Si-XD has also been set in a high-sensitivity CT system and used to measure high-dose-rate X-ray pulses for medical diagnosis (Sato et al., 2013); the amplifier gain should be changed corresponding to the dose rate.

In our research, the major objectives are as follows: to develop a dual-energy detector, to develop a dual current-voltage amplifier, to obtain two-different-energy tomograms simultaneously, and to perform energy-subtraction CT. Therefore, we developed a dualenergy Si-XD (DE-Si-XD) using two Si-XDs and performed Gd-K-edge and energysubtraction imaging.

\section{Experimental methods}

\section{1. $D E-S i-X D$}

Fig. 1 shows a block diagram for detecting X-rays using the DE-Si-XD. The structure of the DE-Si-XD is shown in the same figure, and the DE-Si-XD consists of two SiXDs and a 0.2-mm-thick copper $(\mathrm{Cu})$ filter. In the front $\mathrm{Si}-\mathrm{XD}$, the photocurrents caused by the X-ray absorptions are converted into voltages and amplified using currentvoltage (I-V) and voltage-voltage (V-V) amplifiers [Fig. 1(a)]. To compensate for the image granulation, we used an integrator (Shimamura et al., 2014) with a time constant 
of $50 \mathrm{~ms}$. The integrator is useful for smoothing voltages and has a simple circuit consisting of a $5.0-\mathrm{k} \Omega$ resistor and a $10-\mu \mathrm{F}$ condenser. Subsequently, low-energy X-rays are absorbed by both the front $\mathrm{Si}-\mathrm{XD}$ and the $\mathrm{Cu}$ filter, and the penetrating high-energy $\mathrm{X}$-rays are detected by the back Si-XD. The photocurrents flowing through the back Si$\mathrm{XD}$ are also converted into voltages and amplified using the two amplifiers. The smoothed output voltages from the two integrators are sent to an analog-digital converter (ADC; Contec, AI-1608AY-USB) simultaneously.

The Si-XD (S1087-01, Hamamatsu) has a ceramic substrate with light-receiving dimensions of $1.3 \times 1.3 \mathrm{~mm}^{2}$. The electric capacity between electrodes and the dark current with a bias voltage of $10 \mathrm{~V}$ are $200 \mathrm{pF}$ and $10 \mathrm{pA}$, respectively. The $\mathrm{Cu}$ filter is set between the two Si-XDs, and the DE-Si-XD is covered with an aluminum cap with a 0.2-mm-thick aluminum (Al) window. The detector is shielded using an Al case with a 25- $\mu$ m-thick Al window and a Bayonet Neill Concelman (BNC) connector [Fig. 1(b)].

The circuit diagram of a set of I-V amplifiers is shown in Fig. 2. The bias voltages of the two Si-XDs are both $0 \mathrm{~V}$ to minimize the dark current. In the two amplifiers, the photocurrents $I(t)$ are converted into voltages using a two-channel operational amplifier (LMC662, Texas Instruments), and the output voltages $V_{o}(t)$ as a function of time $t$ are written by:

$$
V_{o}(t)=100 \times 10^{6} \times I(t)
$$

\subsection{DE-CT System}

The DE-CT system is used for obtaining two-different-energy tomograms simultaneously, and an experimental setup of the DE-CT system with the DE-Si-XD is shown in Fig. 3. The CT system consists of an X-ray generator (RXG-0152, R-tec), a 60-mm-stroke linear X-ray scanner (SGSP-26-100, Siguma Koki) with a scan velocity of 25.0-mm/s, and a turntable (SGSP-60YAW-OB, Siguma Koki). The DE-Si-XD with an amplifier module is set on the scanner. The distance between the X-ray source and the detector is $1.00 \mathrm{~m}$, and the distance from the center of turntable to the DE-Si-XD is $45 \mathrm{~mm}$ to decrease the magnification ratio of an object.

The DE-CT is accomplished by repeated linear scans and rotations of the object, and projection curves of the object are obtained by the linear scanning at a tube voltage of 
$90 \mathrm{kV}$ and a current of $1.0 \mathrm{~mA}$. The scanning is conducted in both directions of its movement, and tomograms are reconstructed using the simplest convolution back projection method.

\section{Results}

\subsection{X-ray dose rate}

The X-ray dose rate was measured to infer the skin dose for objects. To measure the dose rate from the $\mathrm{X}$-ray generator, we used an ionization chamber (RAMTEC 1000 plus, Toyo Medic) placed $1.0 \mathrm{~m}$ from the X-ray source (Fig. 4). At a constant tube current of $1.0 \mathrm{~mA}$, the X-ray dose rate increased with the increase in the tube voltage [Fig. 4(a)]. Although the bremsstrahlung X-ray dose rate is proportional to about the second power of the tube voltage at a constant tube current, the dose rate measured was almost in proportion to the tube voltage at a limited voltage range from 75 to $105 \mathrm{kV}$. In contrast, the dose rate was proportional to the tube current at a constant tube voltage of $90 \mathrm{kV}$ [Fig. 4(b)]. The X-ray dose rate was $91.8 \mu \mathrm{Gy} / \mathrm{s}$ at a tube voltage of $90 \mathrm{kV}$ and a current of $1.0 \mathrm{~mA}$.

\subsection{Output voltages}

Fig. 5 shows the output voltages from the front and back Si-XDs measured using a digital multi-meter (ADCE7351E, Advantest). At a constant tube current, the two output voltages from the two Si-XDs increased with increasing tube voltage [Fig. 5(a)]. Subsequently, the two output voltages were in proportion to the tube current at a constant tube voltage [Fig. 5(b)]. In all conditions, the output voltage of the front Si-XD was threefold compared with the back Si-XD. The voltages from the front and back SiXDs were 2.73 and $0.93 \mathrm{~V}$, respectively, at a tube voltage of $90 \mathrm{kV}$ and a current of 1.0 $\mathrm{mA}$.

\subsection{X-ray spectra}

To observe the X-ray spectra entering the front and back Si-XDs, we used a CdTe detector (XR-100T, Amptek) at a tube voltage of $90 \mathrm{kV}$ (Fig. 6). The Gd-K-edge energy 
$50.2 \mathrm{keV}$ is shown in the same figure for reference, and X-ray photons with energies just beyond the K-edge are absorbed effectively by Gd atoms.

The measured spectra entering the front Si-XD and downstream of the front Si-XD and a 0.2-mm-thick $\mathrm{Cu}$ filter are shown in Fig. 6(a). The X-ray spectra detected with the CdTe detector entering the front detector were measured without filtration. The maximum energy of the photons $90 \mathrm{keV}$ corresponding to the tube voltage and the bremsstrahlung peak energy of $35 \mathrm{keV}$ have been observed.

A method for measuring spectra entering the back detector is shown in Fig. 6(b). The single Si-XD was placed just outside the lead pinhole to adjust the center of the Si-XD to the pinhole. Therefore, a 0.2-mm-thick $\mathrm{Cu}$ filter was attached to the $\mathrm{X}$-ray source to infer the spectra entering the back detector. In the previous Fig 6(a), the spectra at the entrance of the back Si-XD filtered by the front Si-XD and the $\mathrm{Cu}$ filter are also presented. By the filtration, the low-energy X-rays were absorbed, and the bremsstrahlung peak energy shifted from 35 to $53 \mathrm{keV}$.

\subsection{Tomography}

To perform tomography, the scan and the rotation steps were $0.5 \mathrm{~mm}$ and $1.0^{\circ}$, respectively, and tomograms were reconstructed with pixel dimensions of $0.5 \times 0.5 \mathrm{~mm}^{2}$. Using this ED-CT system, tomograms are represented as JPEG files, and the maximum and minimum densities correspond to white and black, respectively.

Tomograms of two 15-mm-diameter glass vials filled with Gd media (meglumine gadopentetate) of two different densities are shown in Fig. 7; the densities were 15 and $30 \mathrm{mg} / \mathrm{ml}$, respectively. At a tube voltage of $90 \mathrm{kV}$, the image density difference between the two media increased in the two back-Si-XD images compared with the front image.

The energy-subtraction tomogram $T_{s b}(\mathrm{x}, \mathrm{y})$ for imaging $\mathrm{Gd}$ media was calculated using an equation

$$
T_{s b}(\mathrm{x}, \mathrm{y})=T_{b}(\mathrm{x}, \mathrm{y})-0.5 T_{f}(\mathrm{x}, \mathrm{y})
$$

where $T_{f}(x, y)$ is the front image obtained using the front $\operatorname{Si}-\mathrm{XD}, T_{b}(x, y)$ is the back image using the back Si-XD, and $x$ and $y$ are coordinates. In the $T_{s b}(\mathrm{x}, \mathrm{y})$, the glass vials were subtracted, and Gd media were seen at high contrast. Subsequently, the energysubtraction tomogram $T_{s f}(\mathrm{x}, \mathrm{y})$ for subtracting $\mathrm{Gd}$ media was calculated by an equation 


$$
T_{s f}(\mathrm{x}, \mathrm{y})=T_{f}(\mathrm{x}, \mathrm{y})-0.5 T_{b}(\mathrm{x}, \mathrm{y})
$$

Using this Eq. (3), the Gd media were subtracted, and only the glass vials were imaged.

Fig. 8 shows the image density analysis of the two glass vials filled with Gd media in Fig. 7. In the front-Si-XD image, the image densities of the vials were much higher than the media densities. Compared with the front image, both the image densities and the density difference of the two media increased in the back image. At a tube voltage of 90 $\mathrm{kV}$, the Gd-K-edge imaging was carried out using the back Si-XD.

Fig. 9 shows the result of the tomography of a rabbit-head phantom. The blood vessels of a real rabbit head were filled with gadolinium oxide $\left(\mathrm{Gd}_{2} \mathrm{O}_{3}\right)$ microparticles. The animal operation was carried out in accordance with the animal experiment guidelines of our university. Radiography (angiography) was performed to show tomographic plane using a flat-panel detector (FPD; 1024EV, Rad-icon Imaging) with pixel sizes of $48 \times 48 \mu \mathrm{m}^{2}$. In the front image, the image densities of the bones and the muscles were high, while the image contrast of the thick blood vessels slightly improved in the two back images. Utilizing the energy subtraction, only thick vessels were observed at high contrast using Eq. (2). On the contrary, the vessels were subtracted using Eq. (3).

\section{Discussion}

The output voltage of the front $\mathrm{Si}$-XD was approximately threefold as high as that of the back Si-XD because the low-energy X-rays were absorbed by the front Si-XD and the $\mathrm{Cu}$ filter. The bremsstrahlung peak energy entering the back Si-XD was beyond the Gd-K-edge of $50.2 \mathrm{keV}$. Therefore, the Gd-K-edge CT for imaging the blood vessels could be performed, and only the vessels were observed by the subtraction of the front Si-XD image.

In the DE-CT system, we used two integrators (Shimamura et al., 2014) with time constants of 50 ms to improve image granulation, and the integrator for the back Si-XD was very useful for compensating for the statistical errors caused by the low-dose-rate $\mathrm{X}$-rays. The integrator has a simple circuit consisting of a resistor and a condenser, and the time constant should be maximized corresponding to the scan velocity of the linear scanner. 
The spatial resolutions are primarily determined by the dimensions of the X-rayreceiving area, and the original resolutions were $1.3 \times 1.3 \mathrm{~mm}^{2}$. To improve the spatial resolution, the light-receiving dimensions should be reduced. In addition, the image quality improves with decreasing scan and rotation steps in the CT.

\section{Conclusions}

We developed a DE-Si-XD consisting of two Si-XDs and a 0.2-mm-thick Cu filter, and the effective photon energies detected by the front and back Si-XDs were 33 and 52 $\mathrm{keV}$, respectively; the energy was determined by the half value layer of Al. We also set the DE-Si-XD into a DE-CT system, and two different-energy tomograms were obtained simultaneously to perform energy subtraction and to decrease the statistical errors in the subtraction image.

In the present research, the maximum tube current of the X-ray generator was $2.0 \mathrm{~mA}$, and the DE-CT was carried out at a tube voltage of $90 \mathrm{kV}$ and a current of $1.0 \mathrm{~mA}$. Using this Si-XD, we have already succeeded in measuring high-dose-rate X-ray pulses with tube currents below $0.5 \mathrm{~A}$. Compared with photon-counting detectors, the advantages of the DE-Si-XD are as follows: to detect X-ray beams for medical imaging with tube currents ranging from $0.1 \mathrm{~mA}$ to $1 \mathrm{~A}$, to perform DE-CT without scintillators, and to obtain two-different-energy tomograms simultaneously without energy dispersion. In addition, the disadvantages are as follows: the threshold energy cannot be determined, the structure of the DE-Si-XD is complex, and the Cu filter thickness cannot be changed.

\section{Acknowledgments}

This work was supported by grants from Keiryo Research Foundation, Promotion and Mutual Aid Corporation for Private Schools of Japan, Japan Science and Technology Agency (JST), and Ministry of Education, Culture, Sports, Science and Technology of Japan (MEXT). We also acknowledged the Grant-in-Aid for Strategic Medical Science Research Center from MEXT, 2009-2013 and 2014-2018.

\section{References}

Arakawa, Y., Sato, E., Kogita, H., Hamaya, T., Nihei, S., Numahata, W., Kami, S., Oda, 
Y., Hagiwara, O., Matsukiyo, H., Osawa, A., Enomoto, T., Watanabe, M., Kusachi, S., Sato, S., Ogawa, A., 2014. Investigation of X-ray photon-counting using ceramicsubstrate silicon diode and its application to gadolinium imaging. Jpn. J. Appl. Phys. 53, 072201-1-5.

Feuerlein, S., Roessl, E., Proksa, R., Martens, G., Klass, O., Jeltsch, M., Rasche, V., Brambs, H.J., Hoffmann, M.H.K., Schlomka, J.P., 2008. Multienergy photon-counting K-edge imaging: potential for improved luminal depiction in vascular imaging. Radiology 249, 1010-1016.

Hagiwara, O., Watanabe, M., Sato, E., Matsukiyo, H., Osawa, A., Enomoto, T., Nagao, J., Sato, S., Ogawa, A., Onagawa, J., 2011. Iodine X-ray fluorescence computed tomography system utilizing a cadmium telluride detector in conjunction with a ceriumtarget tube. Nucl. Instr. Meth. A 640, 170-175.

Hyodo, K., Ando, M., Oku, Y., Yamamoto, S., Takeda, T., Itai, Y., Ohtsuka, S., Sugishita, Y., Tada, J., 1998. Development of a two-dimensional imaging system for clinical applications of intravenous coronary angiography using intense synchrotron radiation produced by a multipole wiggler. J. Synchrotron Radiat. 5, 1123-1126.

Matsukiyo, H., Sato, E., Hagiwara, O., Abudurexiti, A., Osawa, A., Enomoto, T., Watanabe, M., Nagao, J., Sato, S., Ogawa, A., Onagawa, J., 2011. Application of an oscillation-type linear cadmium telluride detector to enhanced gadolinium K-edge computed tomography. Nucl. Instr. Meth. A 632, 142-146.

Mori, H., Hyodo, K., Tanaka, E., Uddin-Mohammed, M., Yamakawa, A., Shinozaki, Y., Nakazawa, H., Tanaka, Y., Sekka, T., Iwata, Y., Handa, S., Umetani, K., Ueki, H., Yokoyama, T., Tanioka, K., Kubota, M., Hosaka, H., Ishikawa, N., Ando, M. 1996. Small-vessel radiography in situ with monochromatic synchrotoron radiation. Radiology 201, 173-177. 
Sato, E., Tanaka, E., Mori, H., Kawai, T., Ichimaru, T., Sato, S., Takayama, K., Ido, H., 2004. Demonstration of enhanced K-edge angiography using a cerium target x-ray generator. Med. Phys. 31, 3017-3022.

Sato, E., Tanaka, E., Mori, H., Kawai, T., Inoue, T., Ogawa, A., Yamadera, A., Sato, S., Ito, F., Takayama, K., Onagawa, J., Ido, H., 2005. Variations in cerium x-ray spectra and enhanced K-edge angiography. Jpn. J. Appl. Phys. 44, 8204-8209.

Sato, E., Oda, Y., Abudurexiti, A., Hagiwara, O., Matsukiyo, H., Osawa, A., Enomoto, T., Watanabe, M., Kusachi, S., Sato, S., Ogawa, A., Onagawa, J., 2012. Demonstration of enhanced iodine K-edge imaging using an energy-dispersive $\mathrm{X}$-ray computed tomography system with a $25 \mathrm{~mm} / \mathrm{s}$-scan linear cadmium telluride detector and a single comparator. Appl. Rad. Isot. 70, 831-836.

Sato, E., Oda, Y., Sagae, M., Kodama, H., Hagiwara, O., Matsukiyo, H., Osawa, A., Enomoto, T., Watanabe, M., Kusachi, S., 2013. Pulsed X-ray detection using a directconversion ceramic-substrate silicon diode. Ann. Rep. Iwate Med. Univ. Center Lib. Arts Sci. 49, 1-7.

Shimamura, A., Sato, E., Shikanai, S., Kitakami, K., Nakaya, I., Nishikawa, W., Sato, Y., Yamaguchi, S., Oda, Y., Hagiwara, O., Matsukiyo, H., Enomoto, T., Watanabe, M., Kusachi, S., Ehara, S., 2014. Image-quality improvement in pileup-less cadmiumtelluride X-ray computed tomography using a frequency-voltage converter and its application to iodine imaging. Med. Imag. Inform. Sci. 31, 35-41.

Wang, X., Taguchi, K., Frey, E.C., Meier, D., Wagenaar, D.J., Patt, B.E., 2011. Material separation in X-ray CT with energy resolved photon-counting detectors. Med. Phys. 38, 1534-1541. 


\section{Figure captions}

Fig. 1. Block diagram for performing dual-energy X-ray detection using a DE-Si-XD. (a) Photocurrents flowing through a Si-XD are amplified using I-V and V-V amplifiers, and the output voltages from the $\mathrm{V}-\mathrm{V}$ amplifier are smoothed by an integrator. Low and high energy X-rays are detected by the front and back Si-XDs, respectively. (b) The structure of the DE-Si-XD.

Fig. 2. Circuit diagram of a dual I-V amplifier using a dual operational amplifier chip.

Fig. 3. Experimental setup of a DE-CT system with the DE-Si-XD. DE-CT is accomplished by repeated linear scans and rotations of the object.

Fig. 4. X-ray dose rate measured using an ionization chamber placed $1.0 \mathrm{~m}$ from the Xray source; the size of the data point includes the standard error. (a) Tube voltage dependence at a tube current of $1.0 \mathrm{~mA}$, and (b) tube current dependence at a tube voltage of $90 \mathrm{kV}$.

Fig. 5. Output voltages from the front and back Si-XDs; each data point was obtained using a 5-s-time-constant integrator for smoothing. (a) Variations with the tube voltage at a constant tube current, and (b) variations with the tube current at a constant tube voltage.

Fig. 6. X-ray spectra measured using a CdTe detector at a tube voltage of $90 \mathrm{kV}$. (a) Unfiltered and filtered spectra are exposed to the front and back Si-XDs, respectively. The Gd-K-edge energy of $50.2 \mathrm{keV}$ is shown in the same figure for reference, and $\mathrm{X}$-ray photons with energies just beyond the K edge are absorbed effectively by Gd atoms. (b) A method for roughly measuring X-ray spectra entering the back Si-XD. Low-energy Xray photons are absorbed using a 0.2-mm-thick $\mathrm{Cu}$ filter and a $\mathrm{Si}-\mathrm{XD}$, and penetrating high-energy photons enter the back Si-XD.

Fig. 7. Tomograms of two 15-mm-diameter glass vials filled with two Gd media of different densities. Compared with the front image, the density difference between the two media increased in the two back images. Using Eq. (2), the two vials were almost subtracted, and only two media were observed. In contrast, the two media were subtracted using Eq. (3).

Fig. 8. Image density analysis of the glass vials in Fig. 7 using a program of Image J. (a) Two tomograms obtained using the front and back Si-XDs, and (b) density analysis of 
the two tomograms. Compared with the front image, both the image densities and the density difference of the two media increased in the back image.

Fig. 9. Tomography of a rabbit-head phantom. The blood vessels were filled with $\mathrm{Gd}_{2} \mathrm{O}_{3}$ microspheres. Compared with the front image, the densities of muscles and bones decreased, and the image contrast of the thick vessels improved in the two back images. Thick vessels were seen at high-contrast using Eq. (2), and the vessels were subtracted using Eq. (3). 


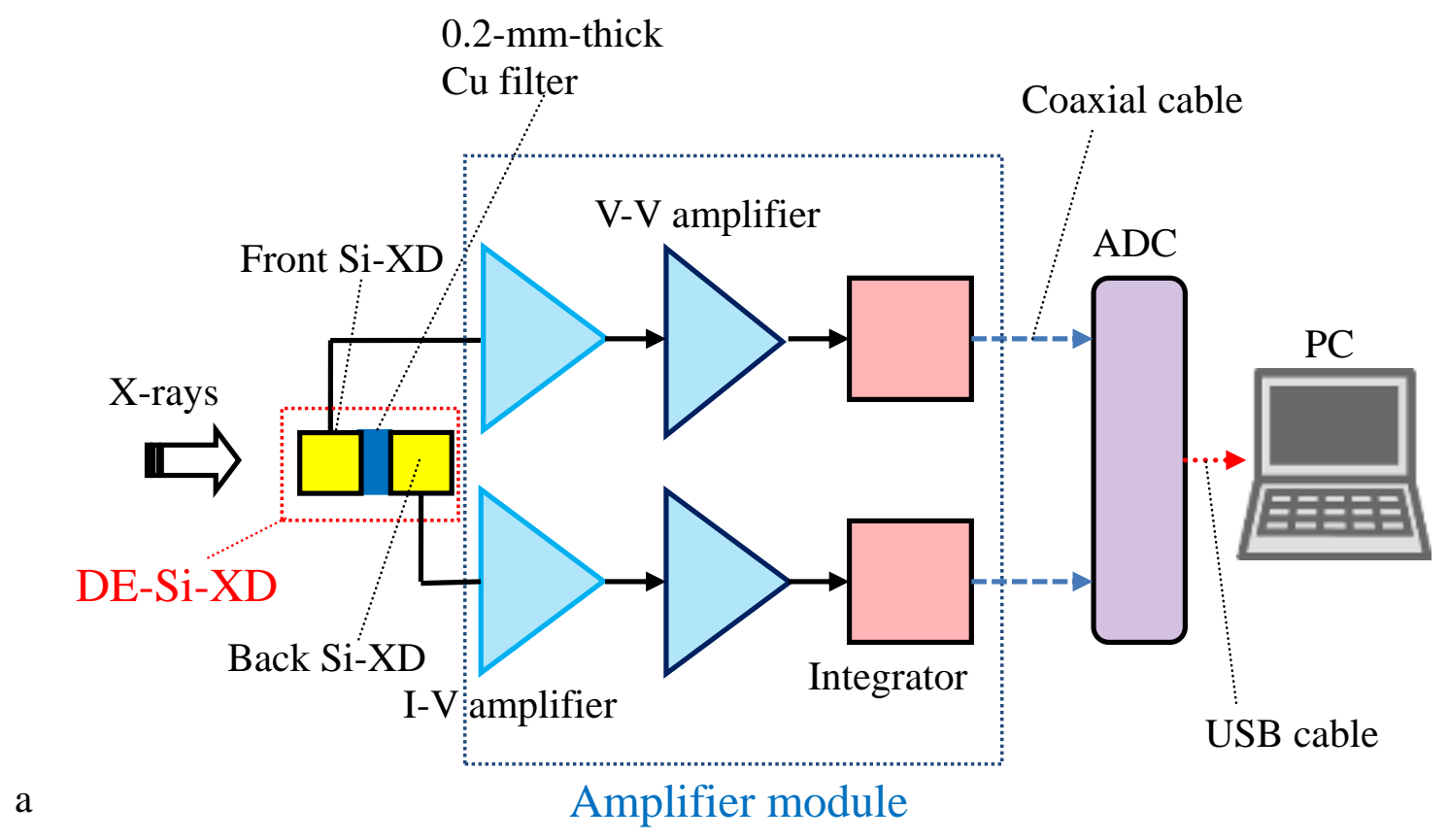

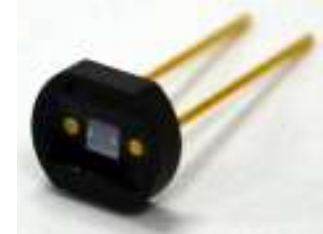

Single Si-XD

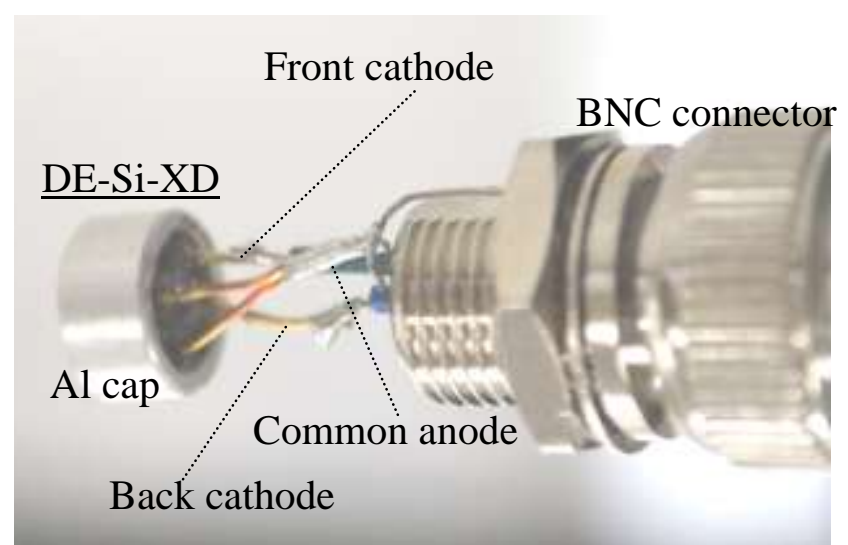

\section{Amplifiers}

Al case

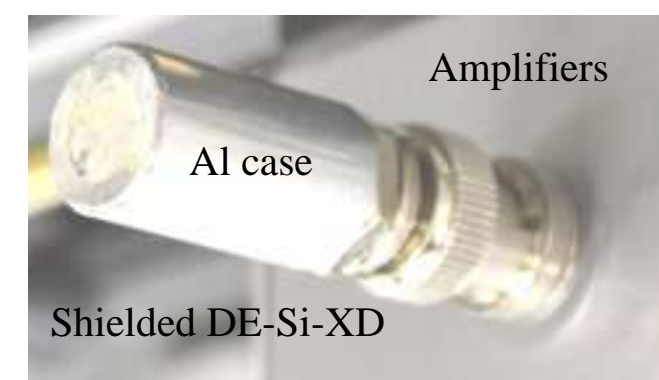

Fig. 1 


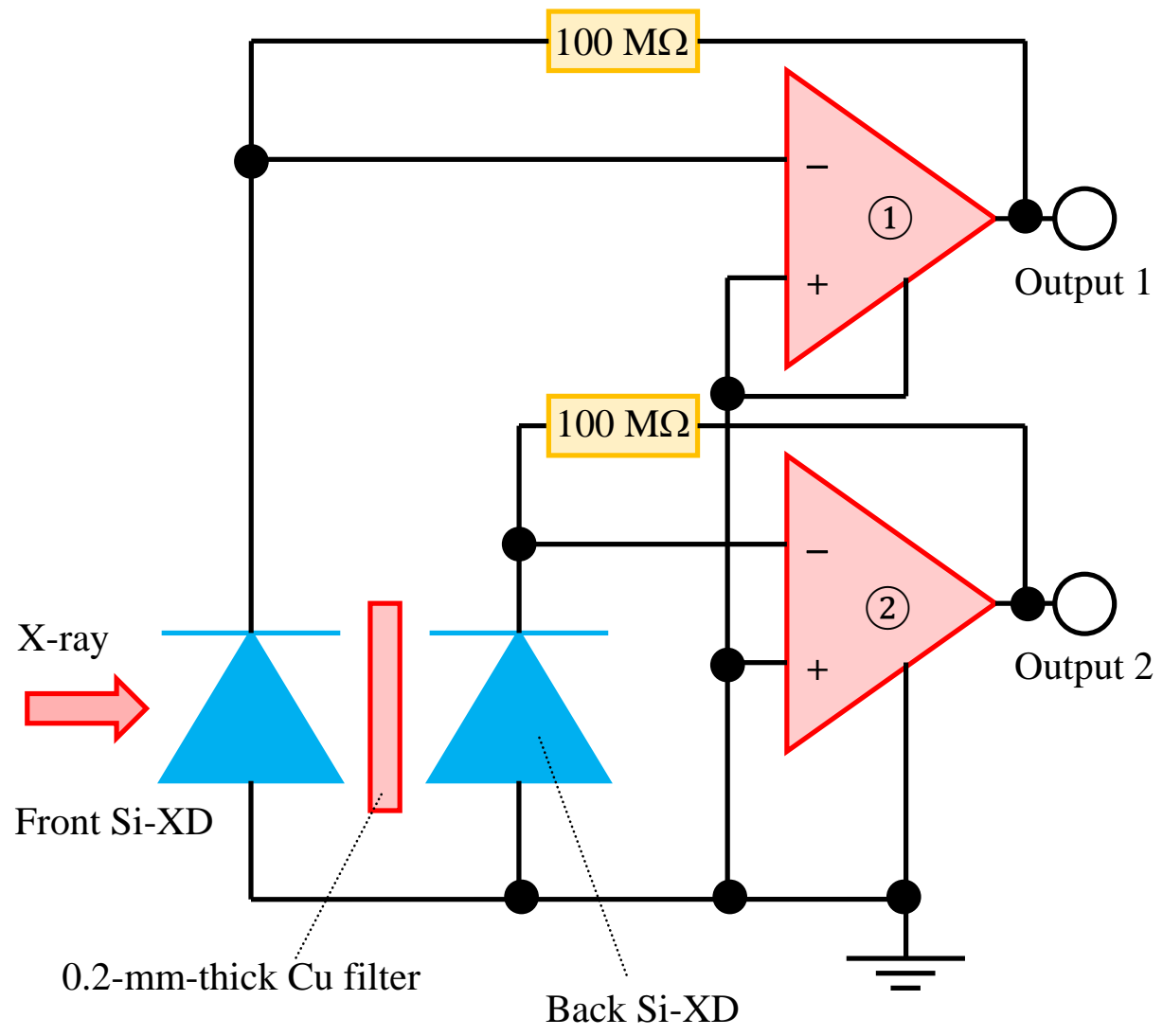

Fig. 2 


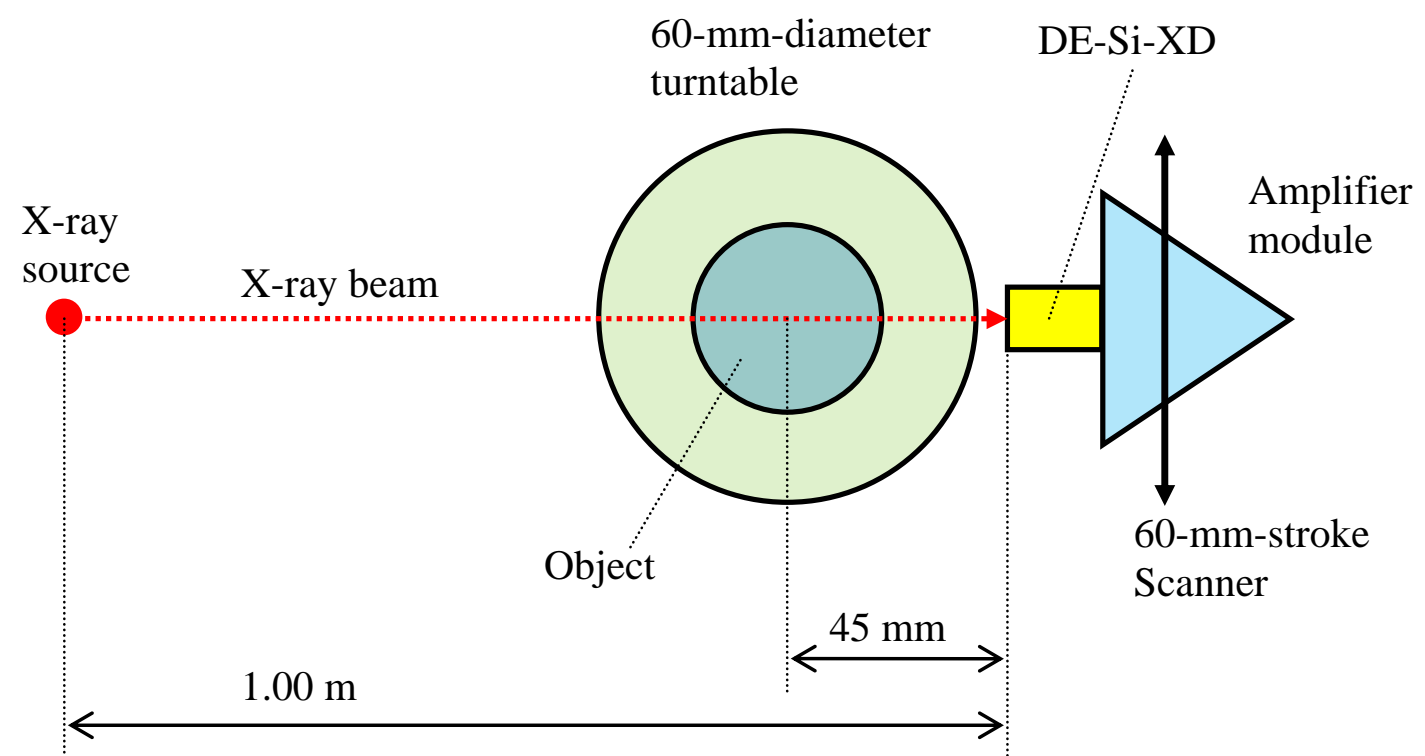

Fig. 3 
a

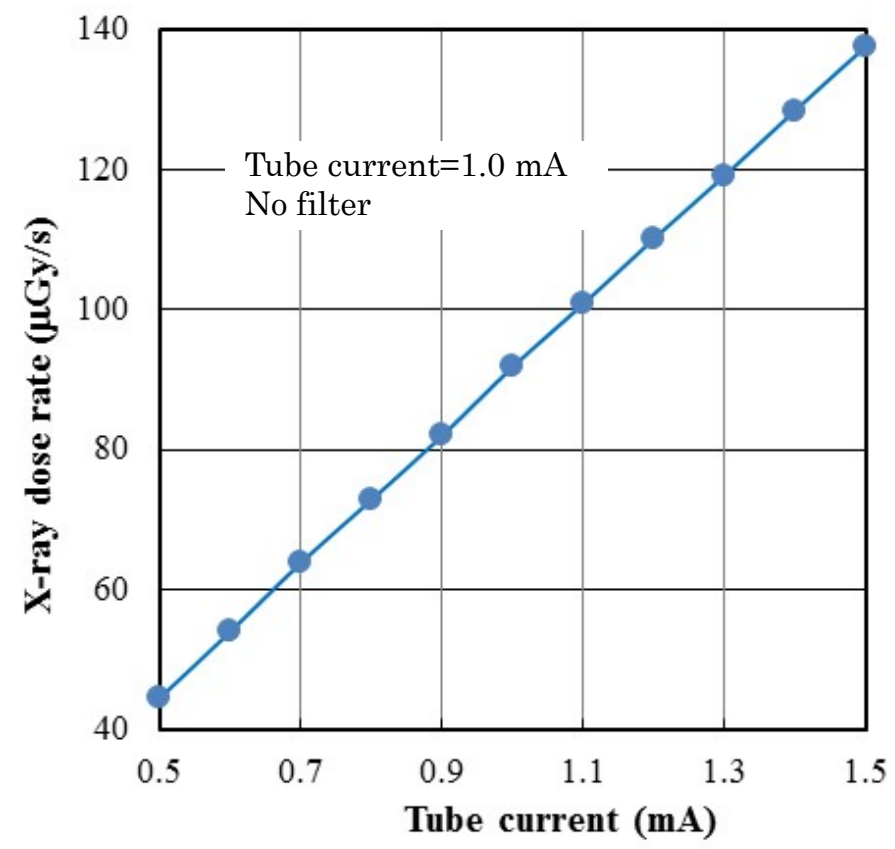

b

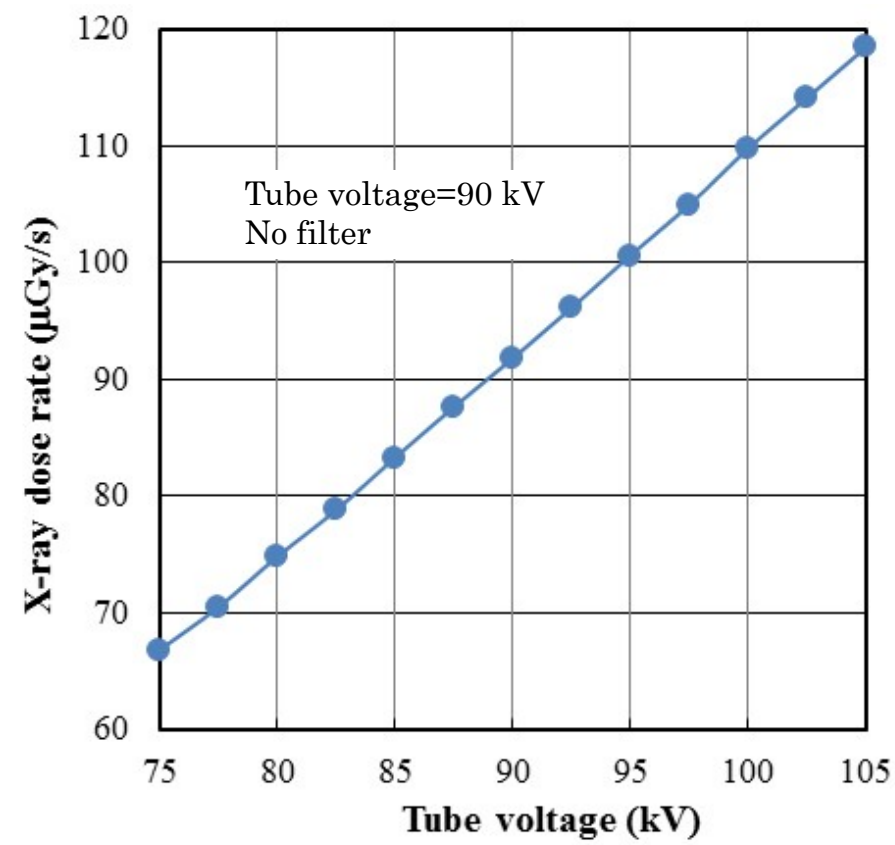

Fig. 4 


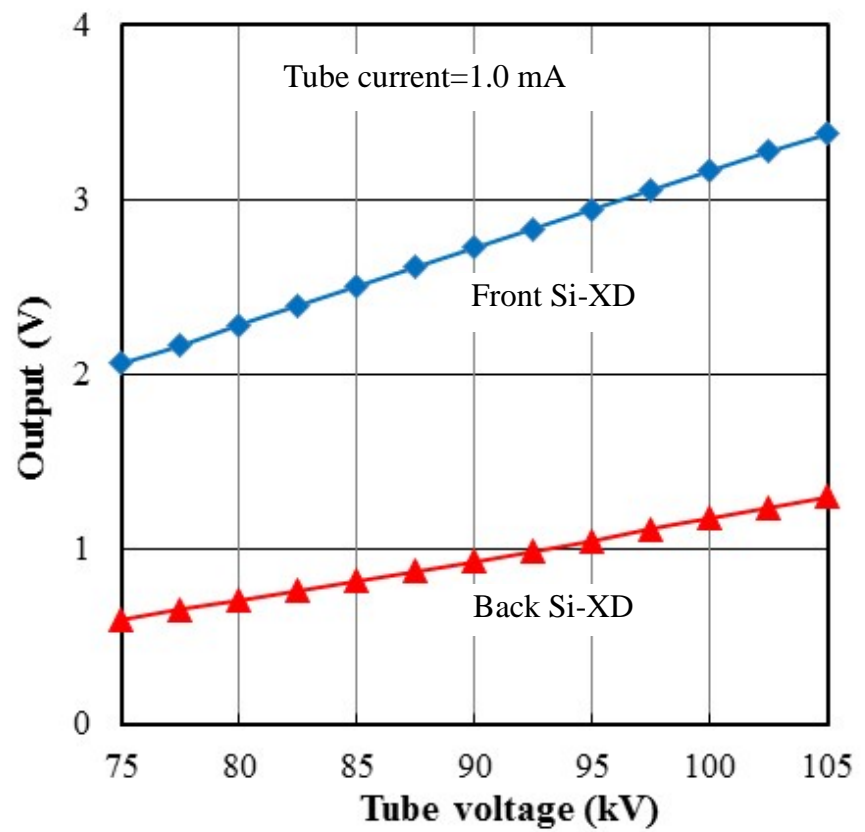

b

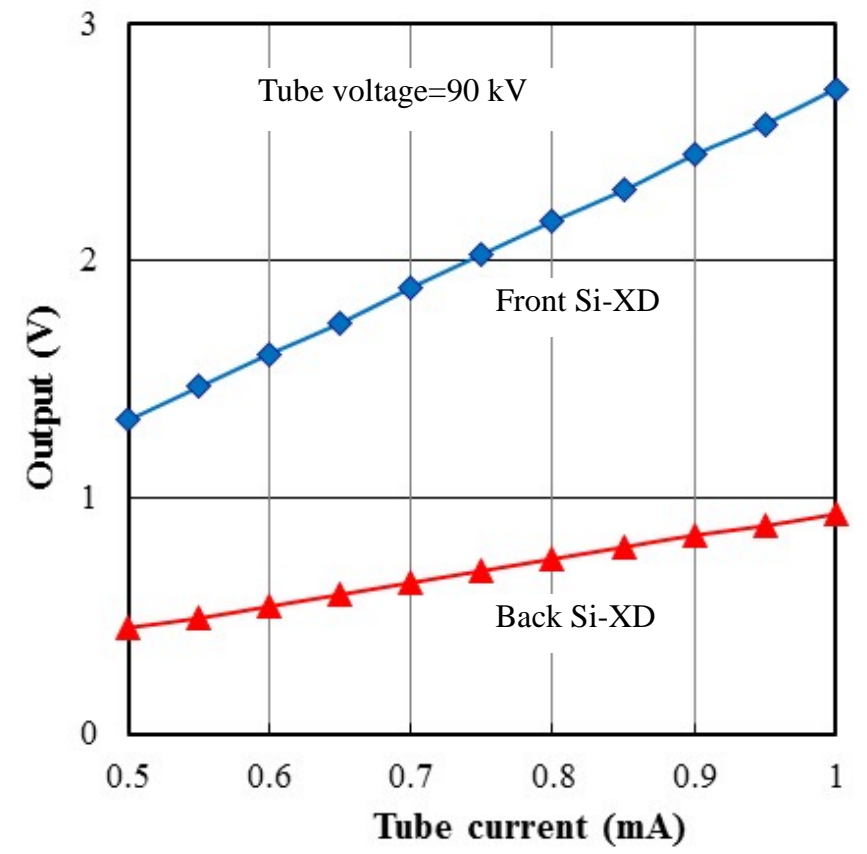

Fig. 5 


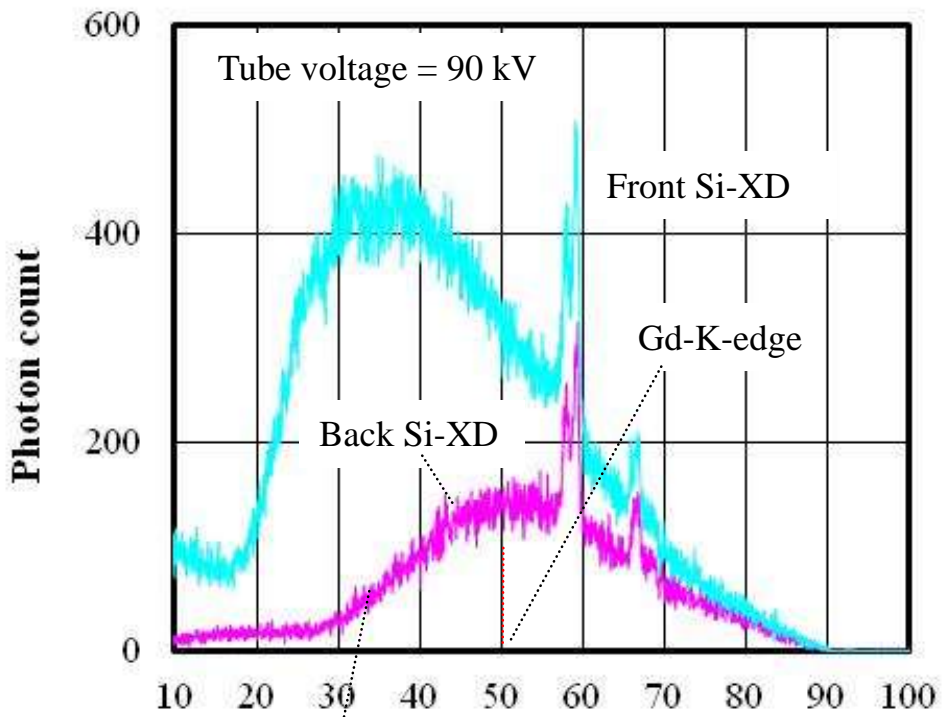

Photon energy (keV)

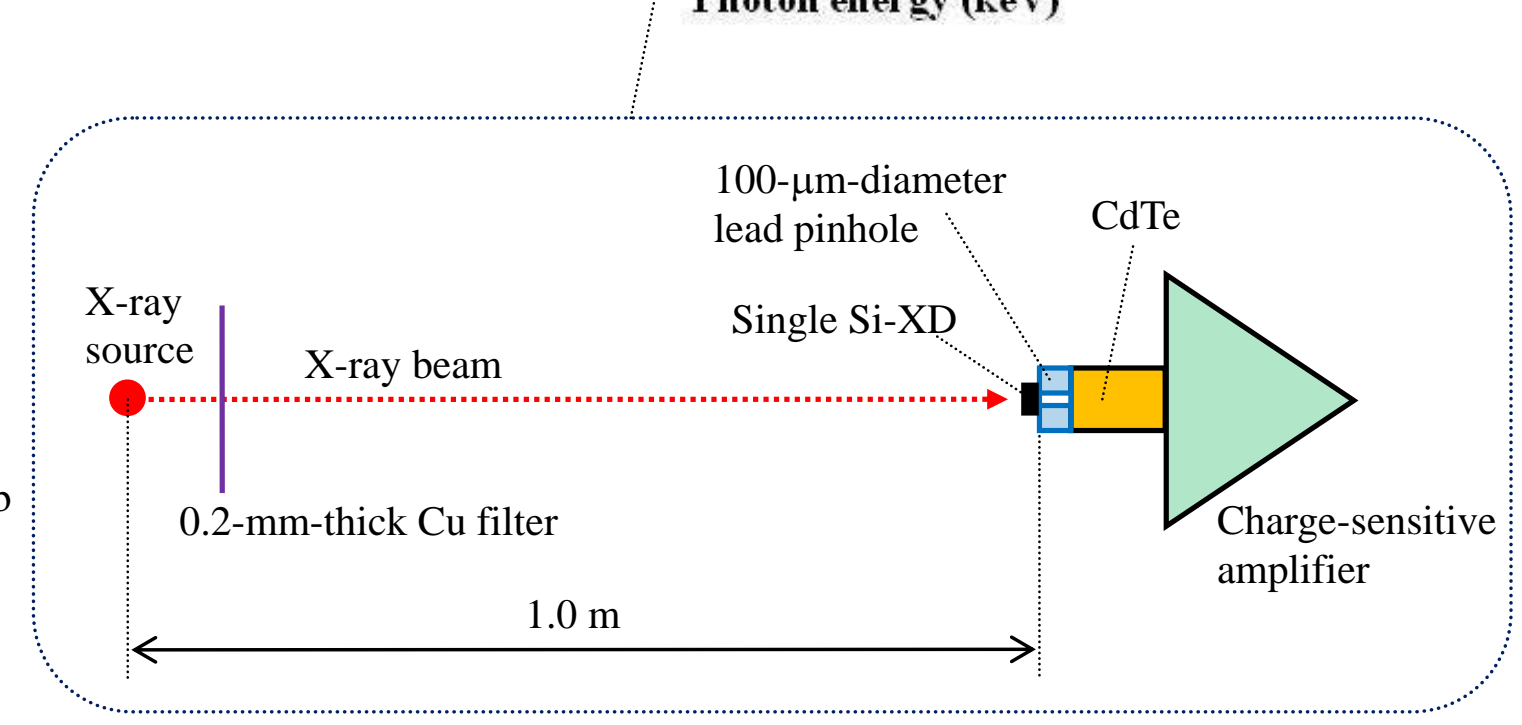

Fig. 6 
(a) $30 \mathrm{mg} / \mathrm{ml} \mathrm{Gd}$

(b) $15 \mathrm{mg} / \mathrm{ml} \mathrm{Gd}$
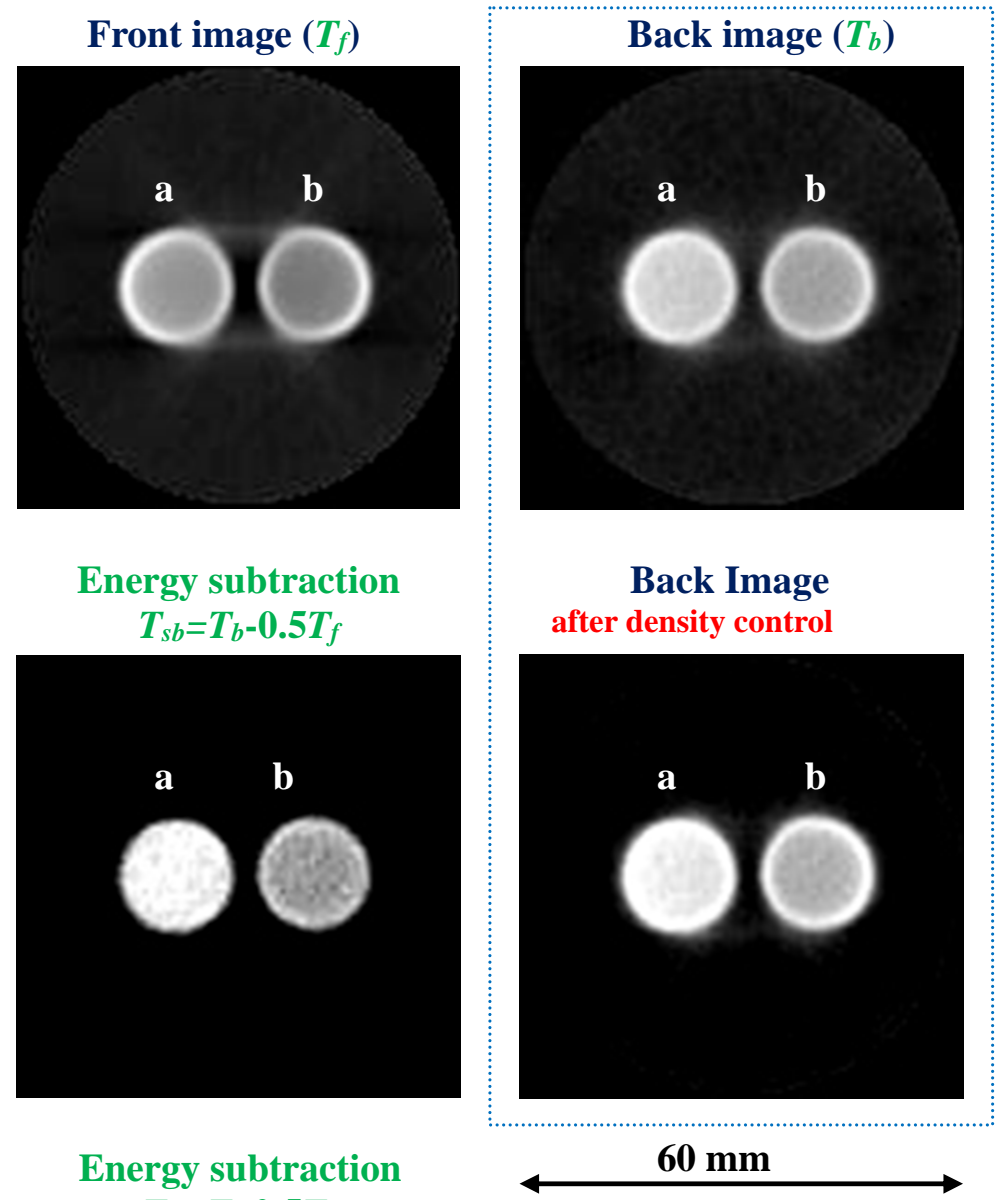

Energy subtraction

$$
T_{s f}=T_{f}-0.5 T_{b}
$$

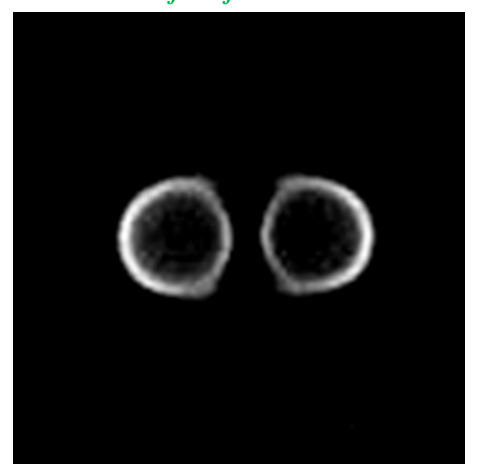

15-mm-diameter glass vials

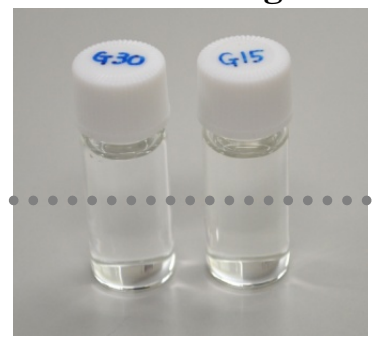

Fig. 7 


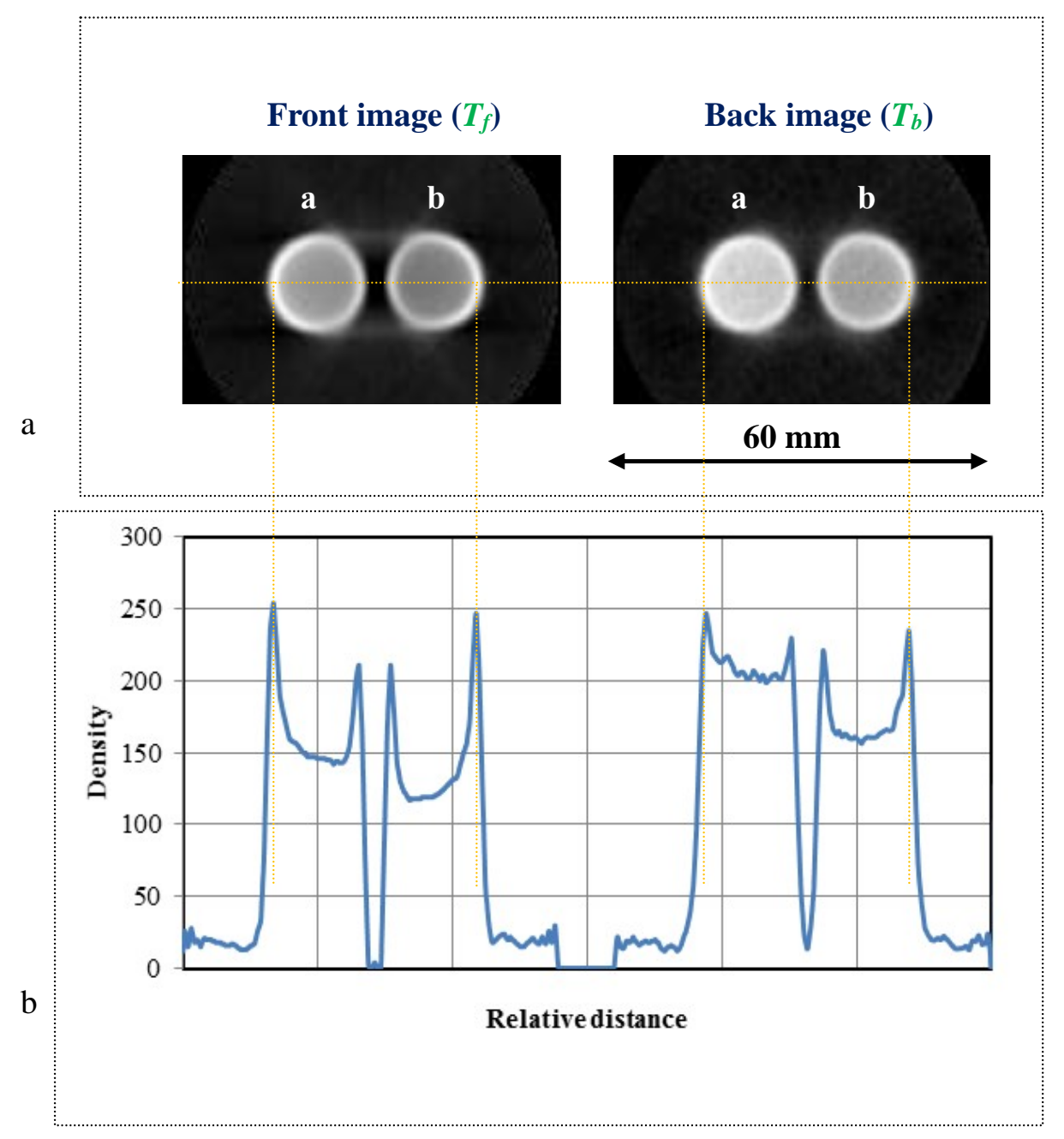

Fig. 8 

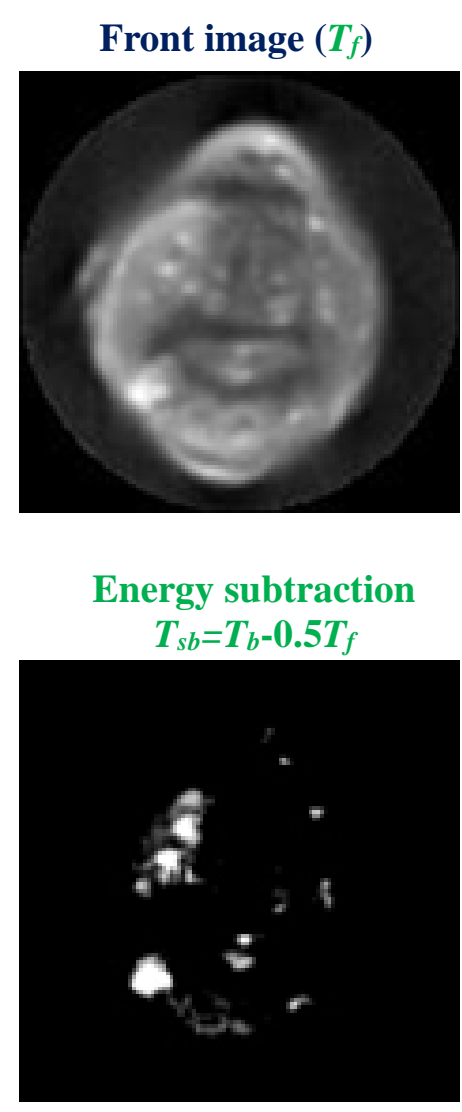

Energy subtraction $T_{s f}=T_{f}-0.5 T_{b}$

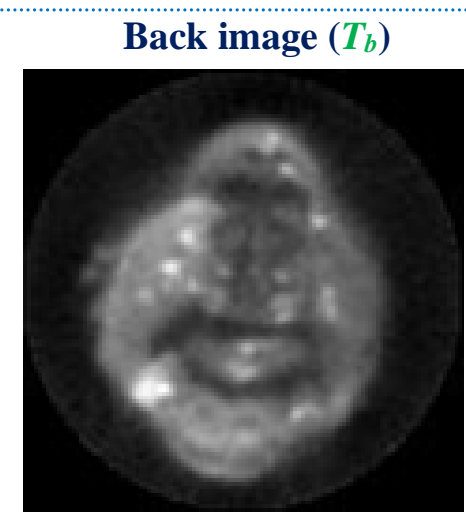

Back Image

after density control

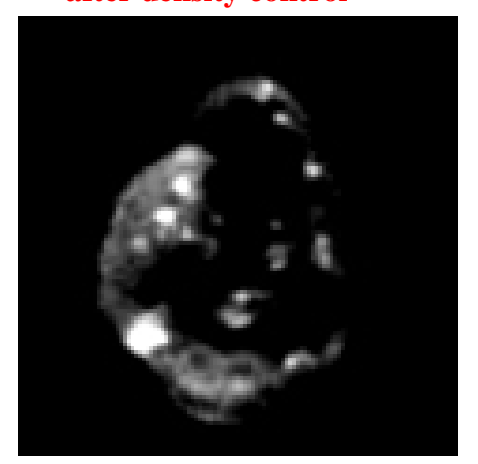

$60 \mathrm{~mm}$
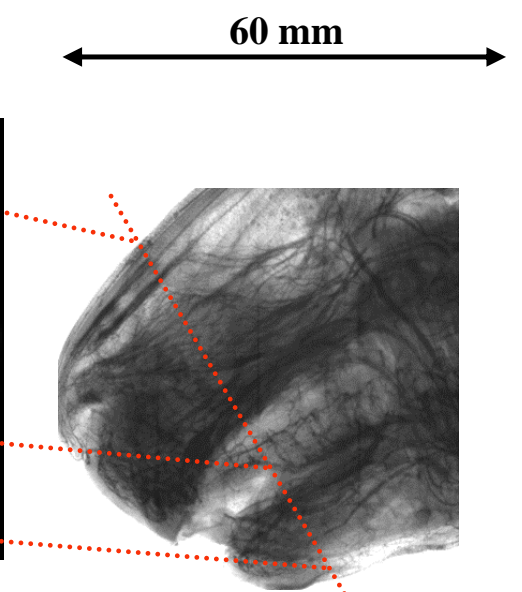

Radiography

Fig. 9 